\title{
CMTM6 knockdown prevents glioma progression by inactivating the mTOR pathway
}

\author{
Li Wei ${ }^{1 \#}$, Qianfeng Wei ${ }^{2 \#}$, Xiaojun Yang ${ }^{1}$, Peng Zhou ${ }^{2}$ \\ ${ }^{1}$ Department of Blood Transfusion, the Third Affiliated Hospital of Soochow University, Changzhou, China; ${ }^{2}$ Department of Neurosurgery, The \\ Third Affiliated Hospital of Soochow University, Changzhou, China \\ Contributions: (I) Conception and design: P Zhou, L Wei, Q Wei; (II) Administrative support: P Zhou; (III) Provision of study materials or patients: \\ L Wei; (IV) Collection and assembly of data: X Yang; (V) Data analysis and interpretation: Q Wei; (VI) Manuscript writing: All authors; (VII) Final \\ approval of manuscript: All authors. \\ \#These authors contributed equally to this work as co-first authors. \\ Correspondence to: Peng Zhou. Department of Neurosurgery, The Third Affiliated Hospital of Soochow University, 185 Juqian Street, Changzhou \\ 213003, China. Email: zpw315@126.com.
}

Background: Gliomas in the adult brain are complicated and aggressive with a poor prognosis. Gene therapy is a recent alternative glioma treatment. We sought to explore the mechanism of chemokine-like factor (CKLF) MARVEL transmembrane domain-containing 6 (CMTM6) in glioma.

Methods: The Cancer Genome Atlas database reports that CMTM6 is expressed in tumors and glioma tissue. CMTM6 expression in glioma tissues and cells was detected and its relationship with clinical pathology was analyzed. Short hairpin ribonucleic acid-CMTM6 lentivirus was transfected into U87 and U251 cells to evaluate malignant glioma cells. Using the biological website (https://string-db.org/cgi/input. pl?Sessionid) and reference retrieval, the pathway that interacted with CMTM6 and related to glioma was identified. The level of the mammalian target of rapamycin pathway-related proteins was detected. Functional rescue experiments were performed using the combination of mTOR activator MHY1485 and the knockdown CMTM6. The growth of xenograft tumors was observed and Ki67-positive expression was determined.

Results: CMTM6 upregulation in gliomas was associated with a poor prognosis. CMTM6 expression was notably higher in gliomas. After the knockdown of CMTM6, the proliferation, invasion, and migration of U87 and U251 cells were inhibited, and the apoptosis rate was increased. Knocking down CMTM6 inactivated the mTOR pathway. The activation of mTOR pathway reversed the inhibitory effects of CMTM6 knockdown on glioma cell behaviors. CMTM6 knockdown reduced tumor volume, body mass, and Ki67-positive expression.

Conclusions: The knockdown of CMTM6 inhibited the activation of mTOR pathway and prevented the malignant episodes of glioma cells.

Keywords: Glioma; CMTM6; mTOR pathway; proliferation; invasion

Submitted Nov 30, 2021. Accepted for publication Jan 27, 2022.

doi: $10.21037 / \mathrm{atm}-21-6894$

View this article at: https://dx.doi.org/10.21037/atm-21-6894

\section{Introduction}

A glioma is a tumor in the central nervous system that originates from glial cells (1). As the most prevalent primary brain tumor, glioma accounts for around $80 \%$ of malignant cases (2). Glioma has high disability and mortality rates due to the high likelihood of metastasis and relapse (3). Generally, high-grade glioma is incurable, and accounts for $8 \%$ of malignant tumors in the human central nervous system (4). At present, the most common regimen for 
glioma is surgical resection plus radiotherapy, or adjuvant chemotherapy (5). However, the survival rate of highgrade glioma patients is extremely low (6). Thus, a better and more effective form of glioma treatment needs to be urgently found.

In recent years, gene therapy has emerged as a new method for glioma treatment. The chemokine-like factor (CKLF) MARVEL transmembrane domain-containing family (CMTM) is ubiquitous in the immune system, cardiovascular system, and tumorigenesis, and the abnormal expression of CMTM is related to the development of various diseases $(7,8)$. A recent in-depth study revealed that CMTM is closely related to the occurrence, development, and metastasis of tumors (9). Among the CMTM family, CMTM6, which is located in the 3p22 region (10), is a regulator of PD-L1 expression and tumor immunity (11). CMTM6 is implicated in epigenetic regulation, embryogenesis, and tumorigenesis (12). It has been reported that high membrane expression of CMTM6 in hepatocellular carcinoma is associated with tumor recurrence (13). CMTM6 promotes cell proliferation and invasion in oral squamous cell carcinoma by interacting with NRP1 (14). Interestingly, CMTM6 overexpression is also related to glioma development (15). However, there is still no specific mechanism of CMTM6 in developing glioma. Studying and determining the mechanism of CMTM6 in glioma may reveal a new way for treating glioma. Additionally, the mammalian target of rapamycin (mTOR) pathway is often activated in tumors, and regulates cell proliferation, apoptosis, and tumor metabolism (16). Phosphatidylinositol 3-kinase (PI3K)-Akt-mTOR pathway is one of the classical cancer-activated signaling pathways in human cancers and the mTOR is considered as a key regulator of PI3K-Akt-mTOR pathway. Glioma growth is driven by pathways that converge on protein synthesis machinery through mTOR signaling molecules (17). The hyperactivation of the mTOR pathway is common in human glioma and critical in monitoring messenger ribonucleic acid (mRNA) translation, and cell biological behaviors (18). Hence, the mTOR pathway plays an essential role in developing of glioma. In our research, based on the above information, we hypothesized that there may be interactions between CMTM6 and the mTOR pathway in glioma progression, innovatively explaining the process of CMTM6 in developing glioma. Thus, we performed histology and molecular experiments to verify this hypothesis and help provide new approaches for glioma treatment.
We present the following article in accordance with the ARRIVE reporting checklist (available at https://atm. amegroups.com/article/view/10.21037/atm-21-6894/rc).

\section{Methods}

\section{Sample collection}

From January 2016 to June 2017, 44 pairs of specimens of glioma and normal tissues were collected from The Third Affiliated Hospital of Soochow University from 24 males and 20 females, aged 26-77 years with an average age of 52 years. According to the World Health Organization (WHO) classification of tumors, there were 16 cases of low-grade glioma (I-II) and 28 cases of high-grade glioma (III-IV).

To be eligible for inclusion in the study, patients had to meet the following inclusion criteria: (I) have been hospitalized for the first time, and not have undergone any radiotherapy, chemotherapy, or immunotherapy before the operation; (II) not have tumors or metastasis in other parts of the body; (III) have undergone surgery by the same group of neurosurgeons under the microscope; (IV) have their tumor tissue pathologically confirmed to be glioma after the operation; and $(\mathrm{V})$ have detailed and complete clinical data for all specimens. All procedures performed in this study involving human participants were in accordance with the Declaration of Helsinki (as revised in 2013). The study was approved by ethics committee board of The Third Affiliated Hospital of Soochow University [No. 2021(135)] and informed consent was taken from all the patients.

\section{Immunobistochemistry}

The glioma and normal tissues were fixed in $4 \%$ paraformaldehyde, embedded in paraffin, and sliced at $3 \mu \mathrm{m}$, and then dewaxed in xylene. Next, the sections were heated in citrate solution for 15 minutes for antigen extraction. After incubation for 10 minutes in 3\% hydrogen peroxide solution to block endogenous peroxidase activity, the sections were blocked in normal goat serum for 20 minutes. Subsequently, the tissue sections were incubated with CMTM6 primary antibody (1:200, NBP1-31183, Novus Biological Inc., Littleton, CO, USA) at $4{ }^{\circ} \mathrm{C}$ overnight, and then with horseradish peroxidase-conjugated antirabbit immunoglobulin G antibody (1:200, NBP2-19301). Afterwards, the sections were dyed with diaminobenzene, counterstained with hematoxylin (Beyotime Biotechnology 
Co., Ltd., Shanghai, China), and sealed by neutral gum. Two senior pathologists read the films in a doubleblind manner, and each section was observed in 5 visual fields under a high-power microscope. According to the cell staining and proportion of positive cells, the mean value was used statistics. In case of disagreement, a $3 \mathrm{rd}$ pathologist was invited to make identification. The $\mathrm{H}$-score semi-quantitative scoring method was used to score immunohistochemical staining results; the $\mathrm{H}$-score ranged from 0 (100\% negative tumor cells) to $300(100 \%$ strongly stained tumor cells), and was calculated using the following formula: ( $\%$ non-staining tumor cells $\times 0)+(\%$ light-yellow tumor cells $\times 1)+(\%$ brown tumor cells $\times 2)+$ $(\%$ chocolate-brown tumor cells $\times 3)(19)$.

\section{HE staining}

The tissue sections were dewaxed, stained with hematoxylin (Beyotime), differentiated with $1 \%$ hydrochloric acid and alcohol for 3 seconds, stained with $1 \%$ eosin for 1 minute, dehydrated, cleared, sealed, and observed under an optic microscope (DM3000, Leica, Solms, Germany).

\section{Cell culture}

The human glioma cell lines (T98G, U87, U251, and U373) and glial cells HEB were provided by the Cell Bank of Type Culture Collection Committee of Chinese Academy of Sciences (Shanghai, China), and cultivated in Dulbecco's modified Eagle's medium (DMEM; Invitrogen, Carlsbad, CA, USA) with $10 \%$ fetal bovine serum (FBS; Sigma, St. Louis, MO, USA) and 1\% penicillin/streptomycin (Sigma) at $37^{\circ} \mathrm{C}$ with $5 \%$ carbon dioxide $\left(\mathrm{CO}_{2}\right)$.

\section{Construction and transfection of interfering lentivirus}

CMTM6 short hairpin RNA (shRNA) was constructed according to the CMTM6 sequence (NCBI Reference Sequence: NM_017801.3) published on the GenBank. The sense sequence was 5 '-gatccGGCCTTCATCTGTGAA GAAGTTGTATTCAAGAGATACAACTTCTTCAC AGATGAAGGCCTTTTTTg-----3', and the antisense sequence was 5'-aattcAAAAAAGGCCTTCATCTGTGA AGAAGTTGTATCTCTTGAATACAACTTCTTCACA GATGAAGGCCg-----3'. CMTM6-shRNA1 was obtained from Shanghai Generay Biotech Co., Ltd. (Shanghai, China) and cloned into pLVX-shRNA lentiviral vector. The recombinant CMTM6-targeting lentivirus (sh-CMTM6) and control mock lentivirus (sh-NC) were transfected into cells.

\section{$R T-q P C R$}

One mL of precooled TRIzol (15596018, Thermo Fisher, Waltham, MA, USA) was added to ground glioma tissues or phosphate buffer saline (PBS)-washed glioma cells for lysis. Trichloromethane was then added to the samples for high-speed centrifugation, and the samples were transferred into the upper aqueous phase. After adding 0.8 volume of isopropanol, the samples were allowed to stand at $-20{ }^{\circ} \mathrm{C}$, and centrifuged at $4{ }^{\circ} \mathrm{C}$ at high speed. Next, $75 \%$ ethanol was added to wash white RNA precipitation, followed by centrifugation and air drying. The RNA was then dissolved with RNA-free double distilled water. After incubation at $55^{\circ} \mathrm{C}$, Nanodrop 2000 (Thermo Fisher) was used to measure the ultra-violet (UV) absorbance of RNA, and the concentration was adjusted to $200 \mathrm{ng} / \mu \mathrm{L}$. According to the Revertaid first strand complementary deoxyribonucleic acid (cDNA) synthesis kit (Toyobo Co., Ltd., Tokyo, Japan), the reverse transcriptase was inactivated on the polymerase chain reaction (PCR) instrument (Applied Biosystems, Carlsbad, CA, USA). The SYBR (Synergy Brands) Green method (Roche Diagnostics, Mannheim, Germany) was used for the quantitative (qPCR). The PCR reaction system included $25 \mu \mathrm{L}$. The PCR conditions were as follows: predenaturation at $95^{\circ} \mathrm{C}$ for 10 minutes, then at $95^{\circ} \mathrm{C}$ for 15 seconds, and then at $60^{\circ} \mathrm{C}$ for 60 seconds, for a total of 40 cycles. The dissolution curve ranged from $60-95^{\circ} \mathrm{C}$, and the temperature rose $0.3^{\circ} \mathrm{C}$ every 15 seconds. The value of each sample was analyzed 3 times to obtain the average value. The CT value was recorded, and the relative expression of mRNA was calculated using the $2^{-\triangle \Delta C T}$ method (20). The primer sequences designed by Sangon Biotech Co., Ltd. (Shanghai, China) are listed in Table 1; GAPDH (glyceraldehyde-3 phosphate dehydrogenase) was used as the control.

\section{Western blot assay}

Following the lysis of the glioma tissue or cells with radio-immunoprecipitation assay buffer (Beyotime), the samples were centrifuged at low temperature to absorb the supernatant and obtain the total protein. After protein concentration measurement by a bicinchoninic acid kit (Pierce, Rockford, IL, USA), equal amounts of proteins were run on $12.5 \%$ sodium dodecyl sulfate-polyacrylamide 
Table 1 Primer sequences for RT-qPCR

\begin{tabular}{lll}
\hline CMTM6 & Forward & 5'-AGTGCCTTTCTTCTGAGTCTCCTT-3' \\
& Reverse & 5'-CTTCAGCCCTAGTGGTATTTTCAG-3' \\
GAPDH & Forward & 5'-CACCATCTTCCAGGAGCGAG-3' \\
& Reverse & 5'-AAATGAGCCCCAGCCTTCTC-3' \\
\hline
\end{tabular}

gel electrophoresis, and transferred to polyvinylidene difluoride membranes. Subsequently, the membranes were probed with primary antibodies against CMTM6 (1/1,000, NBP1-31183), mTOR (mammalian target of rapamycin) (1/1,000, ab32028, abcam), p-mTOR (p-mammalian target of rapamycin) (1/1,000, ab109268, abcam), P70S6K (ribosomal protein S6 kinase) (1/5,000, ab32529, abcam), and p-P70S6K (p-ribosomal protein S6 kinase) (1/500, ab59208, abcam) at $4{ }^{\circ} \mathrm{C}$ overnight. After washing with PBS-Tween (PBST), the membranes were probed with HRP (horseradish peroxidase)-labeled anti-rabbit immunoglobulin G (IgG) antibody (1:5,000, NBP2-19301). After being washed 3 times with PBST, the protein bands were evenly coated with luminescent liquid, and exposed using a full-automatic exposure meter (Fujifilm, Japan). Image J software (Olympus, Japan) was used to analyze the gray value of CMTM6 protein bands.

\section{CCK-8}

Glioma cell suspension $(100 \mu \mathrm{L})$ was prepared in 96-well plates at $2 \times 10^{4}$ cells $/ \mathrm{mL}$. After culturing for 24 hours at $37^{\circ} \mathrm{C}$ with $5 \% \mathrm{CO}_{2}, 10 \mu \mathrm{L}$ of Cell Counting Kit 8 (CCK8) solution (Beijing Wobisen Biotechnology Co., Ltd., Beijing, China) was added to each well at $0,24,48$, and 72 hours. The optical density at $450 \mathrm{~nm}$ was measured using a microplate reader (Bio-Rad, Inc., Hercules, CA, USA).

\section{Flow cytometry}

Cell-cycle detection: the glioma cells were fixed overnight in $70 \%$ ethanol solution $(\mathrm{v} / \mathrm{w})$ at $4{ }^{\circ} \mathrm{C}$, and stained in propidium iodide (PI) the next day at $37^{\circ} \mathrm{C}$ for 30 minutes. The cell cycle was detected on a FACScalibur flow cytometer (BD, Franklin Lakes, NJ, USA), and estimated using Mod Fit LT3.1 software (Verity Software House, Topsham, ME, USA).

Apoptosis detection: after centrifugation, the cells were collected and counted, and $5 \times 10^{5}$ cells were centrifuged at $1,000 \mathrm{rpm}$ at $4{ }^{\circ} \mathrm{C}$ for 10 minutes, and the supernatant was removed. Afterward, $1 \mathrm{~mL}$ of precooled PBS was added to the cells, and the tube slightly shaken. After centrifugation for 2 times at $1,000 \mathrm{rpm}$ at $4{ }^{\circ} \mathrm{C}$, the supernatant was discarded and cells were resuspended in $200 \mu \mathrm{L}$ binding buffer. Next, the cells were mixed with $10 \mu \mathrm{L}$ of annexinV-FITC solution (51-65874X, BD) for 15 minutes without light exposure. Finally, the cells were treated with $300 \mu \mathrm{L}$ of combined buffer and $5 \mu \mathrm{L}$ of PI, and tested on the flow equipment.

\section{Transwell assay}

U87 and U251 cells were trypsinized and resuspended in DMEM containing $1 \%$ FBS, and set at $5 \times 10^{5}$ cells $/ \mathrm{mL}$. Next, $100 \mu \mathrm{L}$ of prepared cell suspension was added to the apical chambers $(8 \mu \mathrm{M})$, which had been precoated with Matrigel (BD), and $600 \mu \mathrm{L}$ of DMEM with $20 \%$ FBS was paved in the basolateral chambers. After 24 hours of culture, the Transwell chambers were removed, and the culture solution in the wells was sucked off, washed twice in calcium-free PBS, and fixed with methanol for 30 minutes. After natural drying, the chambers were dyed for 20 minutes with $0.1 \%$ crystal violet (Beyotime). The uninvaded cells were swabbed lightly with a wet cotton swab, washed with PBS 3 times, and photographed and counted under an inverted light microscope (Leica).

\section{Wound bealing assay}

The cells were cultured in 6 -well microplates at $5 \times 10^{5}$ cells/ well for 24 hours at $37{ }^{\circ} \mathrm{C}$ with $5 \% \mathrm{CO}_{2}$. The cells were then incubated for 4 hours in serum-free DMEM, until cell proliferation slowed down. A sterilized $10-\mu \mathrm{L}$ pipette was used to draw straight lines on the monolayer cells in the microplates, with 3 straight lines between each well. After PBS cleaning, the scratched cells were removed. The microplates were cultured in serum-free DMEM for 24 hours. Finally, the migration distance was observed under an inverted light microscope and analyzed using image-Pro Plus 6.0 software.

\section{Tumorigenicity assay in nude mice}

Specific pathogen-free female immunodeficient mice (3 5 weeks old, 18 21 g) were obtained from Hangzhou Ziyuan Experimental Animal Technology Co., Ltd. [SCXK(Zhejiang)2019-0004]. U87 cells $\left(4 \times 10^{6}\right)$ were transfected with sh-CMTM6 or sh-NC and injected into 
each nude mouse. Tumor growth was measured every 7 days. Tumor volume was calculated as (length $\times$ width $\left.^{2}\right) / 2$. Finally, the mice were euthanized and tumors were removed and weighed. The tumor tissues were incubated with antiKi-67 antibodies (1:500, ab231172, abcam). 3 fields were selected to calculate the ratio of positive tumors and staining intensities. Animal experiments were performed under a project license [No. SYXK(SHU)2017-0044] granted by ethics committee board of The Third Affiliated Hospital of Soochow University, in compliance with Institutional Animal Care and Use Committee (IACUC) guidelines for the care and use of animals.

\section{Statistical analysis}

SPSS 21.0 software (IBM Corp. Armonk, NY, USA) was used for the data processing. The Kolmogorov-Smirnov test was used to check that the data were normally distributed. The measurement data are presented as mean \pm standard deviation. Comparisons between 2 groups were processed by the $t$-test, and comparisons among multi-groups were processed by a 1-way or 2-way analysis of variance (ANOVA), followed by the Tukey multiple comparisons test. The $\mathrm{P}$ value was calculated using a 2 -tailed test, and a $\mathrm{P}$ value $<0.05$ indicated a significant difference.

\section{Results}

\section{CMTM6 was upregulated in glioma and associated with a} poor prognosis

The overexpression of CMTM6 is related to the development of glioma (13). Through The Cancer Genome Atlas (TCGA) database (http://ualcan.path.uab. edu/analysis.html), we learned that CMTM6 expression was upregulated in various cancers (see Figure 1A), and CMTM6 upregulation was associated with a poor prognosis in glioma (see Figure 1B,1C); however, CMTM6 expression did not differ significantly across each age group (see Figure 1D). Hematoxylin and eosin (HE) staining and immunohistochemistry were performed on normal tissues, and low-/high-grade glioma tissues. The density of cells in the normal brain tissues was uniform, the layers were clear, the nuclei were regular without abnormal staining in cytoplasm and heteromorphic cells. Conversely, the density of low-grade glioma cells increased, the cytoplasm was stained, and the nuclei were stained deeply, while the cells in high-grade glioma gathered in different sizes and were in disorder, and the heteromorphism was obvious (see Figure 1E). CMTM6 was higher in high-grade glioma tissue than low-grade glioma tissue $(\mathrm{P}<0.05)$ (see Figure $1 F$ ). The real-time-qPCR (RT-qPCR) results showed that CMTM6 expression in glioma tissues was higher than that in adjacent normal tissues (see Figure 1G). The average CMTM6 expression detected by RT-qPCR (21) was used as the threshold value, and the patients were assigned to low expression $(\mathrm{n}=21)$ and high expression groups $(\mathrm{n}=23)$, and the relationship between CMTM6 expression and clinicopathology was analyzed. CMTM6 expression in glioma tissue was correlated with WHO grade $(\mathrm{P}<0.05)$, but CMTM6 expression was not significantly correlated with other clinicopathological parameters (including gender, age, and tumor diameter) (see Table S1). Additionally, CMTM6 expression in glioma cells was higher than that in normal glial cells $(\mathrm{P}<0.05$; see Figure $1 H)$.

\section{Knockdown of CMTM6 prevented glioma cell proliferation and promoted apoptosis}

To further explore the effect of CMTM6 on glioma cells, U87 and U251 cells were transfected with sh-CMTM6 (see Figure 2A,2B). The CCK- 8 results revealed that the proliferation of cells decreased markedly after CMTM6 knockdown $(\mathrm{P}<0.05$; see Figure $2 \mathrm{C})$. The flow cytometry results showed that after the knockdown of CMTM6, the apoptosis rate and the G1/G0 phase increased significantly $(\mathrm{P}<0.01)$ (see Figure 2D,2E).

\section{Knockdown of CMTM6 inbibited the invasion and migration of glioma cells}

The Transwell and wound healing assays indicated that after the knockdown of CMTM6, the invasion and migration of U87 and U251 cells was noticeably inhibited $(\mathrm{P}<0.01$; see Figure $3 A, 3 B$ ).

\section{Knockdown of CMTM6 blocked the activation of mTOR patbway}

From the above results, we noted that the knockdown of CMTM6 inhibited the malignant biological behaviors of glioma. We then sought to understand the downstream mechanism of CMTM6 affecting glioma. A biological website (https://string-db.org/cgi/input.pl? Sessionid) revealed that CMTM6 and mTOR were interrelated (see Figure $4 A$ ). The inhibition of the mTOR pathway inhibited 

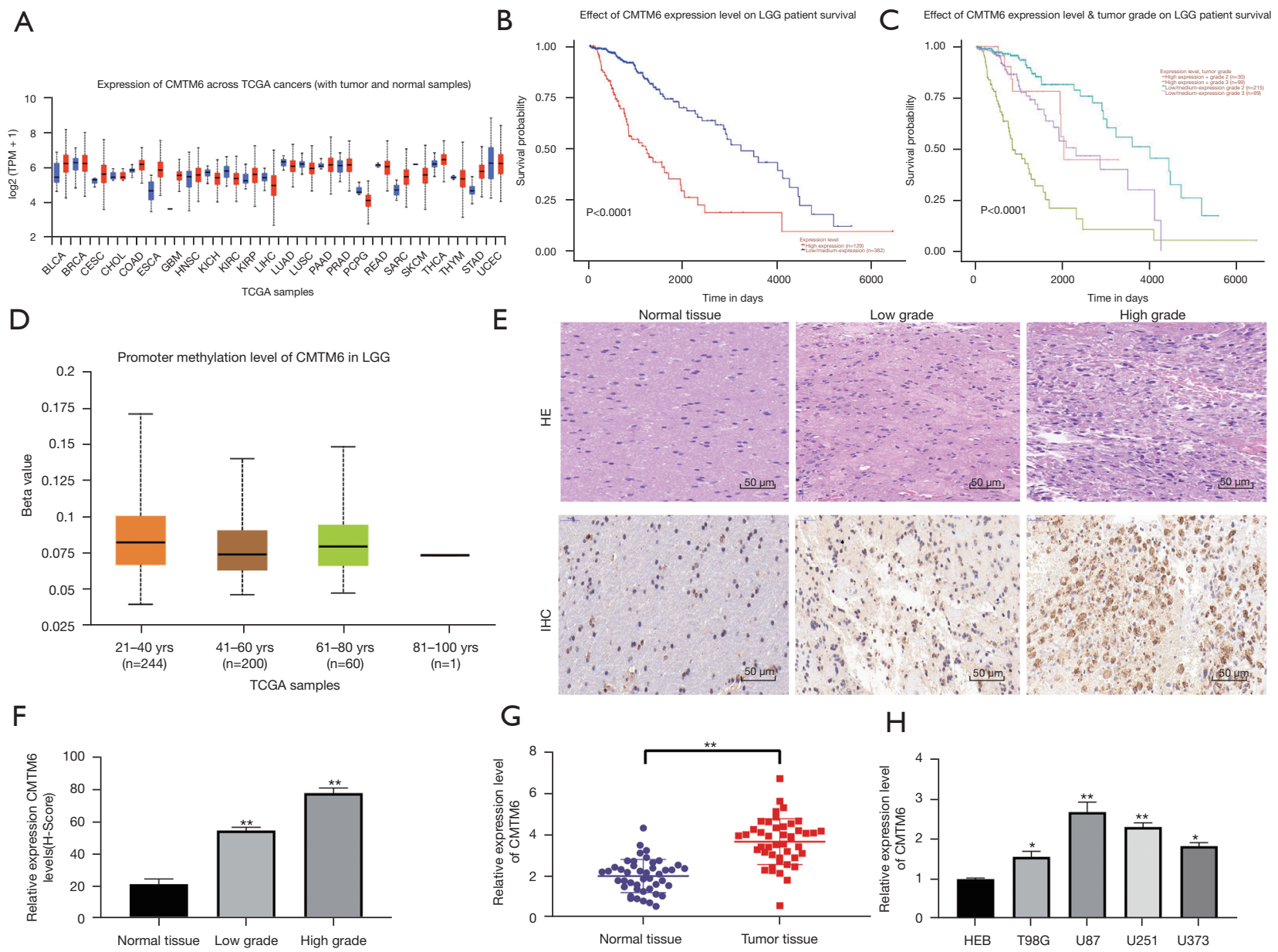

Figure 1 CMTM6 was upregulated in glioma and correlated with a poor prognosis. (A) TCGA database showed that CMTM6 was upregulated in various tumor tissues; blue indicates normal tissues, and red indicates tumor tissues; (B) TCGA database showed that the upregulation of CMTM6 was related to a poor prognosis; (C) TCGA database showed the relationship between the survival prognosis of patients with different grades of glioma and CMTM6 expression; (D) TCGA database showed CMTM6 expression in glioma patients with different ages; (E) normal tissue and glioma tissue were stained with HE and immunohistochemistry; (F) relatively positive expression of CMTM6 measured by immunohistochemistry; (G) CMTM6 expression in tumor tissues and normal tissues as detected by RT-qPCR (n=44); (H) CMTM6 expression in glioma cells and glial cells as detected by RT-qPCR ( $n=3)$. The data in Panel G were analyzed by $t$-test, and data in Panels F/H were analyzed by a 1-way ANOVA and Tukey's multiple comparisons test. * $, \mathrm{P}<0.05,{ }^{* *}, \mathrm{P}<0.01$. TCGA, The Cancer Genome Atlas.

the proliferation and invasion of glioma cells (22). Thus, we speculated that CMTM6 may affect glioma development via the mTOR pathway. To verify this hypothesis, we detected the levels of mTOR pathway-related proteins in U87 and U251 cells by Western blot, and found that $\mathrm{p} / \mathrm{t}-\mathrm{mTOR}$ and $\mathrm{p} / \mathrm{t}-\mathrm{p} 70 \mathrm{~S} 6 \mathrm{~K}$ decreased significantly after CMTM6 knockdown $(\mathrm{P}<0.05$; see Figure $4 B, 4 C)$.

\section{Activation of mTOR patbway reversed the inbibitory effects of CMTM6 knockdown on glioma cell behaviors}

We conducted functional rescue experiments to further confirm that the knockdown of CMTM6 inhibited the malignant episodes of glioma cells by inhibiting the mTOR pathway. The cells were treated with the mTOR activator, 

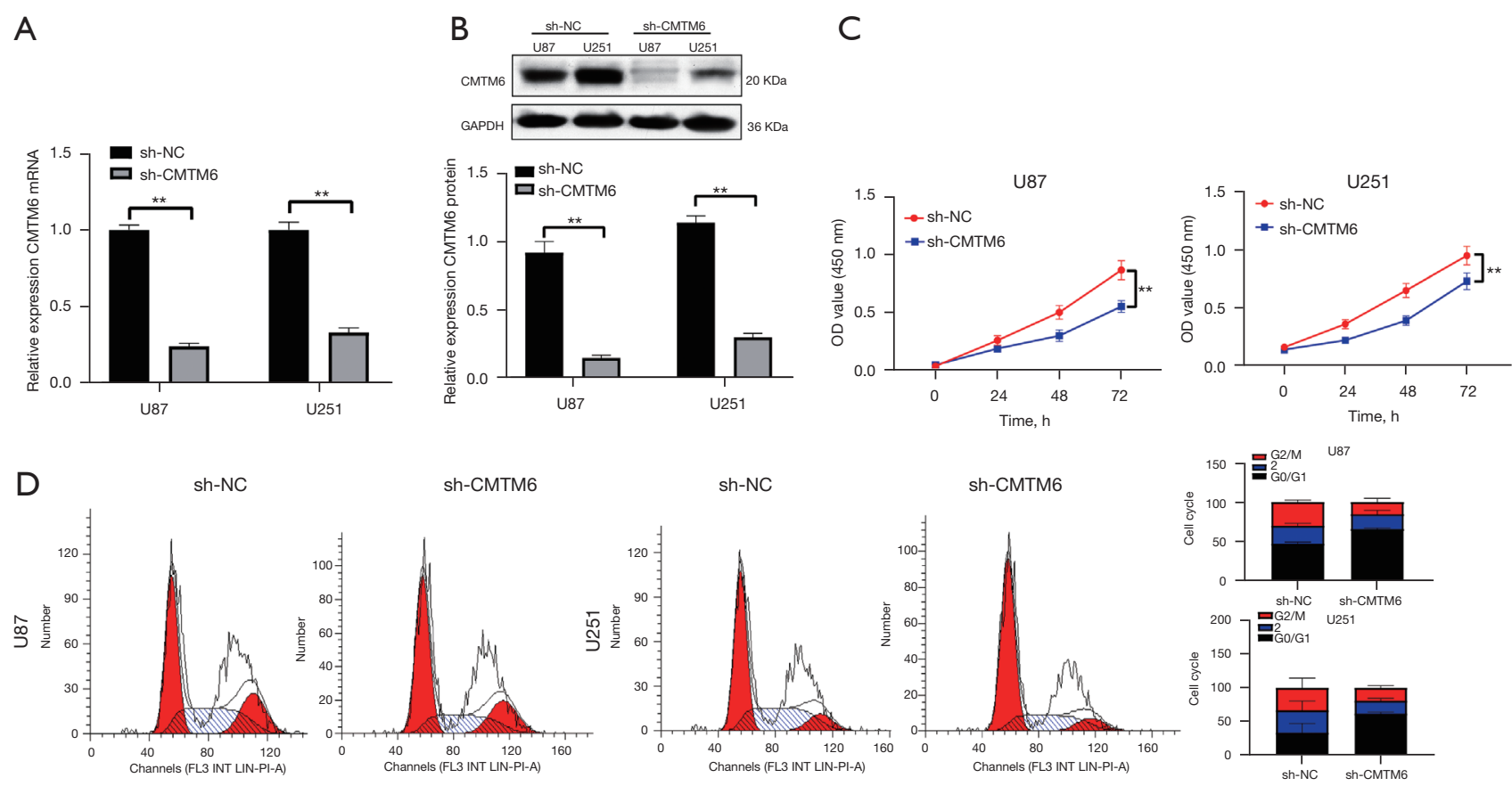

$E$
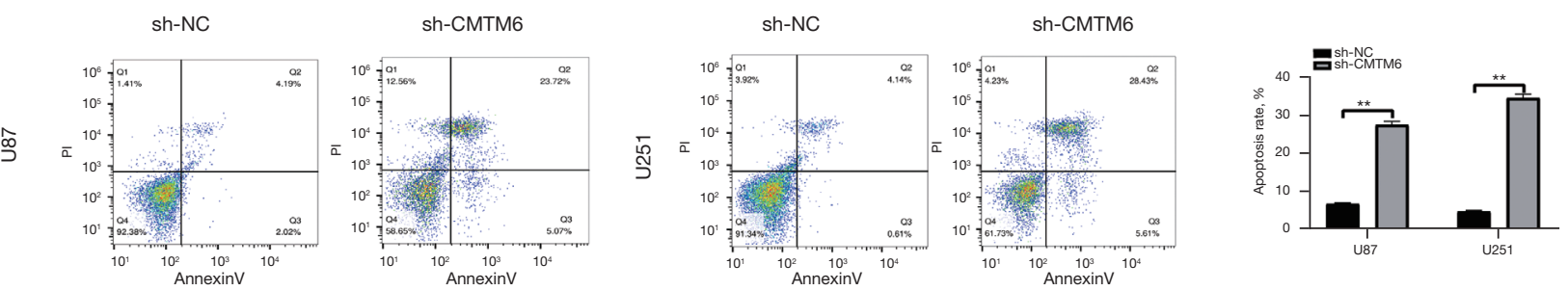

Figure 2 The knockdown of CMTM6 inhibits proliferation and promotes apoptosis of glioma cells. (A,B) RT-qPCR and Western blot detected CMTM6 levels in glioma cells after the transfection of sh-CMTM6; (C) CCK-8 detected the proliferation of glioma cells at 0, 24, 48, and 72 h, respectively; (D) flow cytometry detected the apoptosis rate of glioma cells; (E) flow cytometry detected the cell-cycle distribution. Replicates $=3$; all data were analyzed by a 2 -way ANOVA and Tukey's multiple comparisons test. ${ }^{* *}, \mathrm{P}<0.01$.

MHY1485, and knocked down with CMTM6. The biological behaviors of U87 and U251 cells were evaluated by CCK-8 (see Figure 5A), flow cytometry (see Figure 5B), Transwell (see Figure 5C), and wound healing assays (see Figure 5D). The proliferation, invasion, and migration of U87 and U251 cells in the sh-CMTM6 + MHY1485 group were promoted and apoptosis was inhibited compared to that caused by the single knockdown CMTM6 (all $\mathrm{P}<0.05$ ).

\section{Knockdown of CMTM6 inbibited glioma cell growth in vivo}

U87 cells stably transfected with sh-CMTM6 were injected subcutaneously into nude mice, and the volume and mass of tumors and Ki67-positive expression were measured. After CMTM6 knockdown, the volume and mass of tumors decreased (see Figure 6A-6C), and the positive expression of Ki67 was decreased significantly as the immunohistochemistry results indicated (all $\mathrm{P}<0.05$; see Figure 6D).

\section{Discussion}

Glioma is the most common tumor in the central nervous system, and accounts for nearly $27 \%$ of all brain tumors and $80 \%$ of all malignant primary brain tumors (23). The molecular mechanism of glioma is complicated, and urgently needs to be understood to improve antitumor efficacy and overall survival (24). In this study, we discussed the mechanism underlying CMTM6 in glioma. Consequently, a novel molecular interaction was identified in which CMTM6 knockdown prevented glioma progression by inactivating the mTOR pathway. 

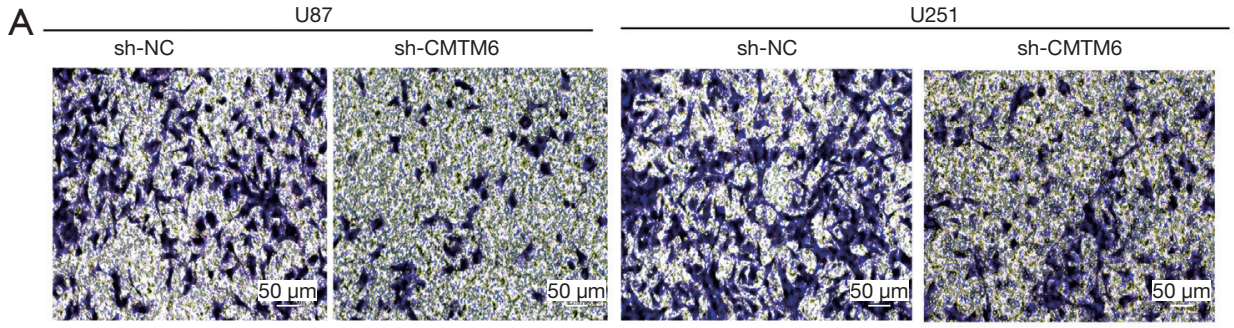

B

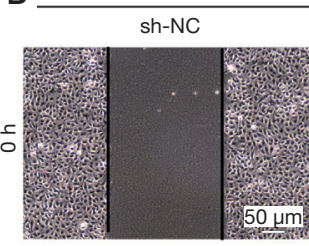

U87
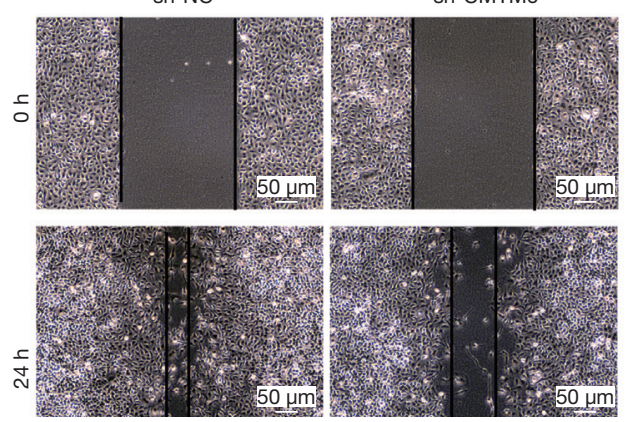

U251
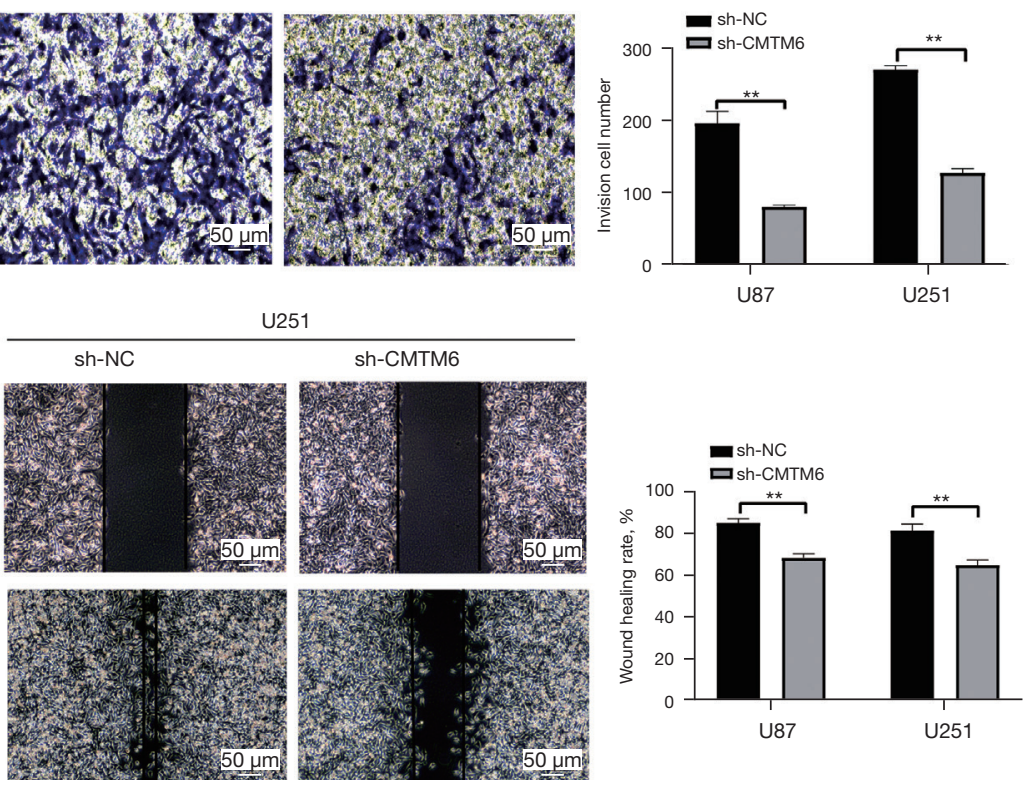

Figure 3 The knockdown of CMTM6 inhibits he invasion and migration of glioma cells. (A) The invasion ability of glioma cells detected by Transwell assay dyed with $0.1 \%$ crystal violet (Beyotime). Scale bar $=50 \mu \mathrm{m}$; (B) the migration ability of glioma cells detected by wound healing assay. Replicates $=3$; all the data were analyzed by a 2 -way ANOVA and Tukey's multiple comparisons test. ${ }^{* *}, \mathrm{P}<0.01$. Scale bar $=50 \mu \mathrm{m}$.

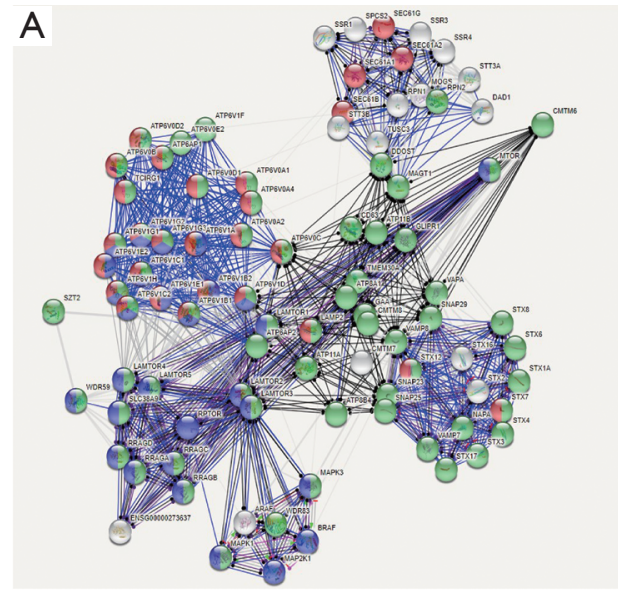

B
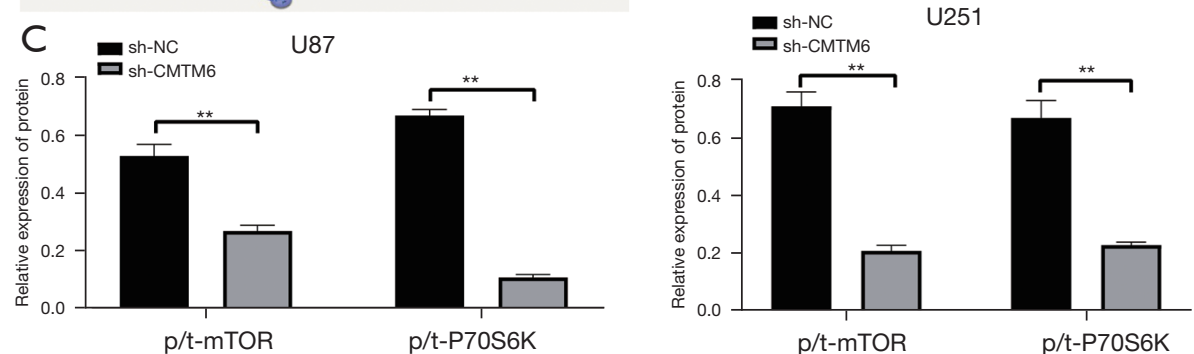

Figure 4 The knockdown of CMTM6 inhibits the activation of mTOR pathway. (A) A website (https://string-db.org/cgi/input.pl?sessionid) predicted that CMTM6 and mTOR had a mutual relationship; (B) Western blot detected the expression of mTOR pathway-related proteins; (C) quantitative analysis histogram of protein bands. Replicates $=3$; The data in panel $(\mathrm{C})$ were analyzed by a 2-way ANOVA and Tukey's multiple comparisons test. ${ }^{* *}, \mathrm{P}<0.01$. mTOR, mammalian target of rapamycin. 

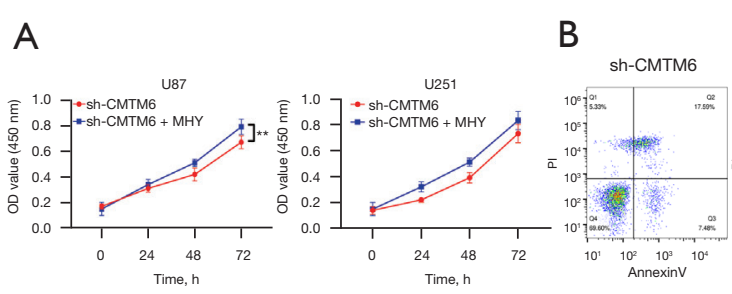

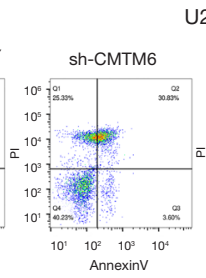

U251

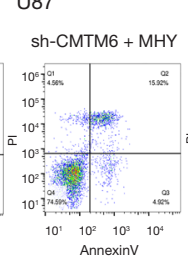

C

U87
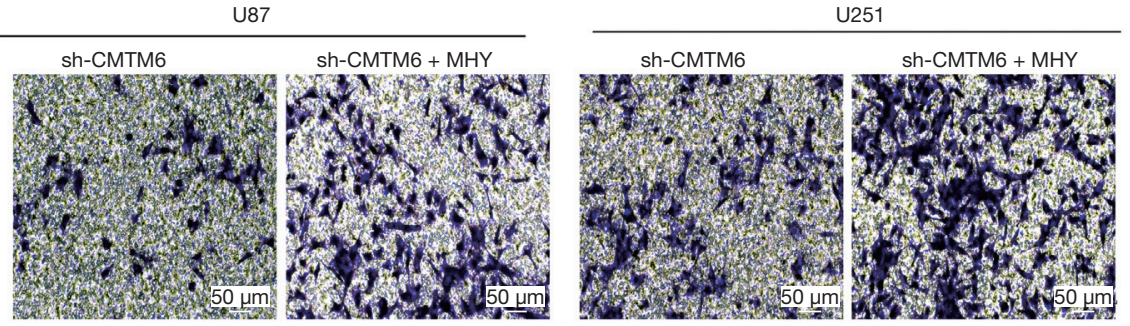

U87
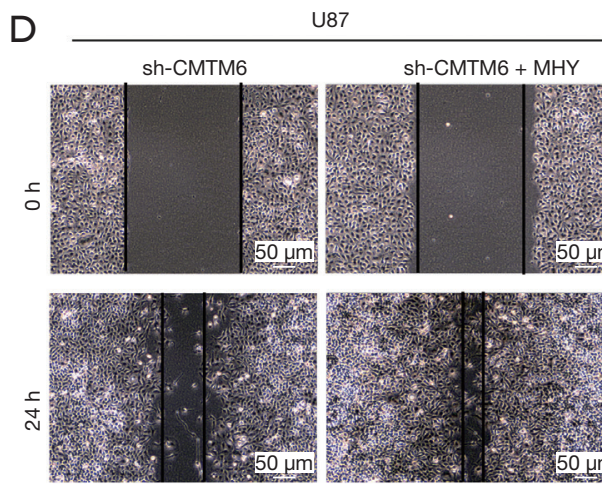
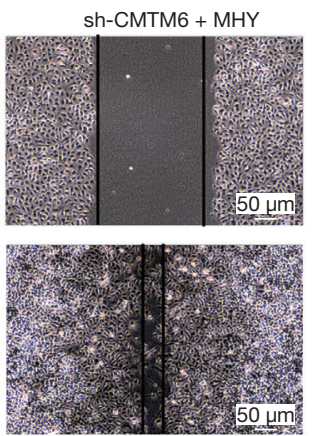
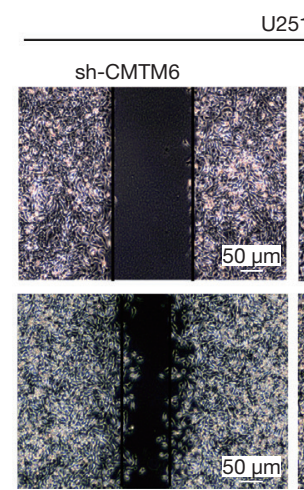

$\mathrm{U} 251$
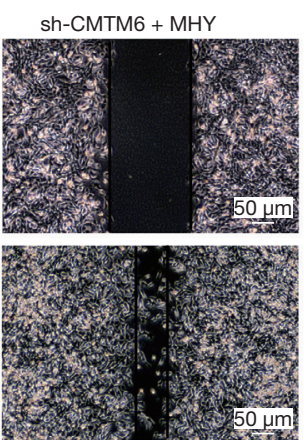
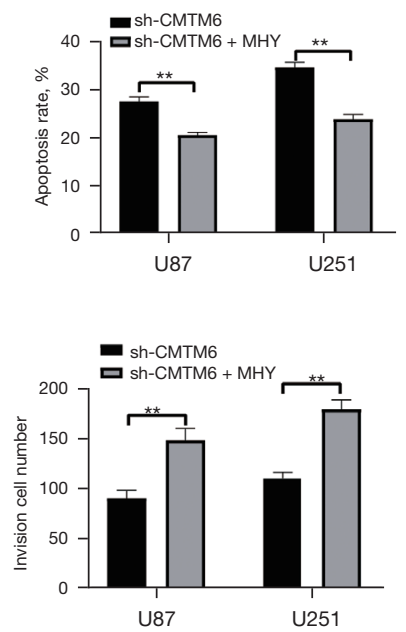

Figure 5 The knockdown of CMTM6 inhibits proliferation and promotes apoptosis of glioma cells. (A) CCK-8 detected the proliferation of glioma cells; (B) flow cytometry detected the apoptosis rate; (C) Transwell assay detected the invasion ability of glioma cells dyed with $0.1 \%$ crystal violet (Beyotime). Scale bar $=50 \mu \mathrm{m}$; (D) wound healing assay detected the migration ability of glioma cells. Replicates =3; the data were analyzed by a 2-way ANOVA and Tukey's multiple comparisons test. ${ }^{* *}, \mathrm{P}<0.01$. Scale bar $=50 \mu \mathrm{m}$.

A previous study found that high CMTM6 expression is related to reduced survival time and high-grade malignant gliomas (15). TCGA database revealed CMTM6 expression was upregulated in various cancers. In this study, through histological observation and RT-qPCR detection, we observed that CMTM6 was higher in high-grade glioma tissues and cells and correlated with severe pathological deterioration and WHO grade. CMTM6 is an important regulator in the tumor microenvironment and anti-tumor activities (25). CMTM6 overexpression indicates a poor prognosis for patients with head and neck squamous cell carcinoma (11).

To further evaluate the biological effect of CMTM6 on glioma cells, U87 and U251 cells were transfected with sh-CMTM6. After the knockdown of CMTM6, the malignant episodes of glioma cells were inhibited. CMTM6 is a leading modulator of $\mathrm{T}$ lymphocyte-mediated anti- tumor responses (25). CMTM6-deficient tumor cells are susceptible to antigen-specific cytotoxic T-lymphocytes and result in longer survival in murine transplantable melanoma models (26). In the in-vivo experiments, after CMTM6 knockdown, the volume and mass of tumors and Ki67positive expression were significantly decreased. Similarly, CMTM6 deficiency has been shown to enhance T cell activation and the anti-tumor response in mouse melanoma models (27).

Recent research on the glioma found that several deregulated pathways converge and activate mTOR (28). The biological website predicted that CMTM6 and mTOR were interrelated. This led us to speculate that CMTM6 may affect glioma development via the mTOR pathway. The Western blot results revealed that $\mathrm{p} / \mathrm{t}-\mathrm{mTOR}$ and p/t-p70S6K in U87 and U251 cells decreased significantly after CMTM6 knockdown. mTOR is frequently activated 
A

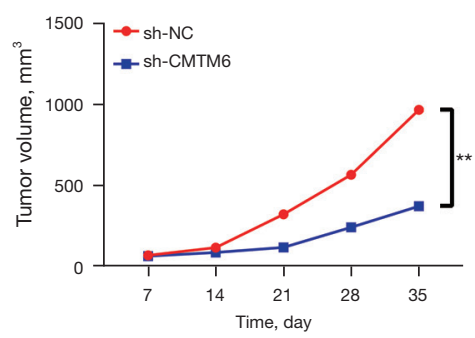

C
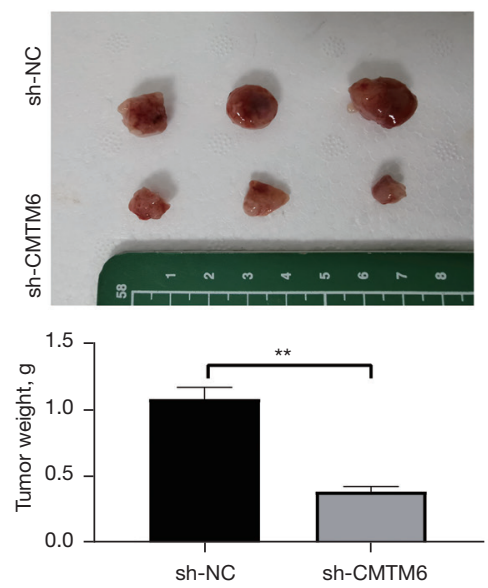

B

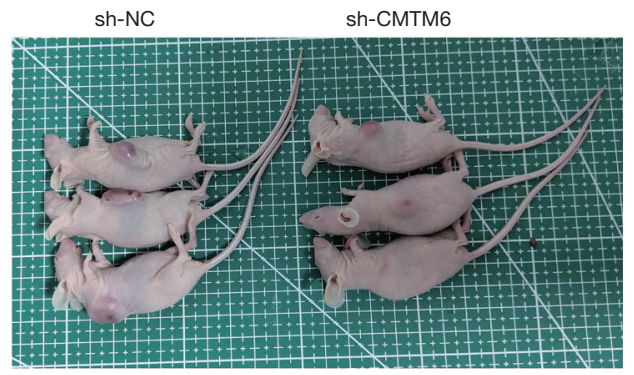

sh-NC
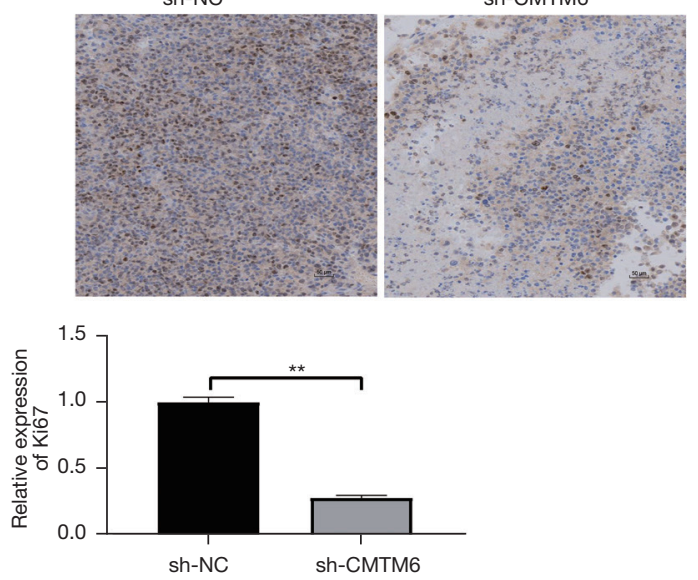

Figure 6 The knockdown of CMTM6 inhibits the growth of glioma cells in vitro. (A) Measurement of tumor volume (n=3); (B,C) measurement results of tumor mice and body mass on the 35 th day $(n=3)$; (D) immunohistochemical staining detected the positive expression of $\mathrm{Ki} 67(\mathrm{n}=3)$. The data in panels (C,D) were tested by $t$-test, and the data in panel (A) were analyzed by a 2 -way ANOVA and Tukey's multiple comparisons test. ${ }^{* *}, \mathrm{P}<0.01$.

in pediatric low-grade glioma (29). The radio-sensitizing effects of mTOR inhibitors have been identified in the glioma xenografts in mice (30). The cells were treated with a mTOR activator (MHY1485) and CMTM6 was knocked down to further confirm that CMTM6 suppressed the malignant episodes of glioma cells by inhibiting the mTOR pathway. As expected, the malignant biological behaviors of glioma cells were inhibited in the sh-CMTM6 + MHY1485 group. The activation of mTOR pathway is related to prooncogenic cell processes; thus, mTOR could be used as an underlying target in a novel combined regimen (31). The inhibition of mTOR induces glioma-activated microglia to differentiate into the M1 phenotype with cytotoxic activity to prevent M2 phenotype, which promotes tumor growth (28). Innovative combination therapy with mTOR inhibitors will contribute to the application of mTOR targeted drugs and the personalized treatment of cancer patients (32).

In conclusion, the present study showed that CMTM6 knockdown prevented glioma progression by inactivating the mTOR pathway. Our findings provide novel insights for glioma treatment and lay the foundation for further study about the underlying mechanism of CMTM6 in other tumors. However, this study had some limitations (e.g., the sample size was small). More efforts should be made to improve the relevant experiments in the future.

\section{Acknowledgments}

Funding: This work was supported by the Changzhou Science and Technology Program (Grant No. CJ20210083), the Postdoctoral Research Fund of Jiangsu Province (Grant No. 2021K242B), the Major Science and Technology Project of Changzhou Municipal Health Commission (Grant No. ZD202005), and the Young Talent Science and Technology Project of Changzhou Municipal Health Committee (Grant No. QN202024). 


\section{Footnote}

Reporting Checklist: The authors have completed the ARRIVE reporting checklist. Available at https://atm. amegroups.com/article/view/10.21037/atm-21-6894/rc

Data Sharing Statement: Available at https://atm.amegroups. com/article/view/10.21037/atm-21-6894/dss

Conflicts of Interest: All authors have completed the ICMJE uniform disclosure form (available at https://atm. amegroups.com/article/view/10.21037/atm-21-6894/coif). The authors have no conflicts of interest to declare.

Ethical Statement: The authors are accountable for all aspects of the work in ensuring that questions related to the accuracy or integrity of any part of the work are appropriately investigated and resolved. All procedures performed in this study involving human participants were in accordance with the Declaration of Helsinki (as revised in 2013). The study was approved by ethics committee board of The Third Affiliated Hospital of Soochow University [No. 2021(135)] and informed consent was taken from all the patients. Animal experiments were performed under a project license [No. SYXK(SHU)2017-0044] granted by ethics committee board of The Third Affiliated Hospital of Soochow University, in compliance with Institutional Animal Care and Use Committee (IACUC) guidelines for the care and use of animals.

Open Access Statement: This is an Open Access article distributed in accordance with the Creative Commons Attribution-NonCommercial-NoDerivs 4.0 International License (CC BY-NC-ND 4.0), which permits the noncommercial replication and distribution of the article with the strict proviso that no changes or edits are made and the original work is properly cited (including links to both the formal publication through the relevant DOI and the license). See: https://creativecommons.org/licenses/by-nc-nd/4.0/.

\section{References}

1. Ghotme KA, Barreto GE, Echeverria V, et al. Gliomas: New Perspectives in Diagnosis, Treatment and Prognosis. Curr Top Med Chem 2017;17:1438-47.

2. Khalil BD, El-Sibai M. Rho GTPases in primary brain tumor malignancy and invasion. J Neurooncol 2012;108:333-9.
3. Wang C, Kang L, Wang X, et al. Expression of miR-200a and chemotherapeutic treatment efficacy of glioma. Oncol Lett 2018;15:5767-71.

4. Chen J, McKay RM, Parada LF. Malignant glioma: lessons from genomics, mouse models, and stem cells. Cell 2012;149:36-47.

5. Chen X, Mao YG, Yu ZQ, et al. Potential rules of anesthetic gases on glioma. Med Gas Res 2020;10:50-3.

6. Taylor LP. Diagnosis, treatment, and prognosis of glioma: five new things. Neurology 2010;75:S28-32.

7. Duan HJ, Li XY, Liu C, et al. Chemokine-like factor-like MARVEL transmembrane domain-containing family in autoimmune diseases. Chin Med J (Engl) 2020;133:951-8.

8. Huang ZM, Li PL, Yang P, et al. Overexpression of CMTM7 inhibits cell growth and migration in liver cancer. Kaohsiung J Med Sci 2019;35:332-40.

9. Wu K, Li X, Gu H, et al. Research Advances in CKLFlike MARVEL Transmembrane Domain-containing Family in Non-small Cell Lung Cancer. Int J Biol Sci 2019;15:2576-83.

10. Mamessier E, Birnbaum DJ, Finetti P, et al. CMTM6 stabilizes PD-L1 expression and refines its prognostic value in tumors. Ann Transl Med 2018;6:54.

11. Chen L, Yang QC, Li YC, et al. Targeting CMTM6 Suppresses Stem Cell-Like Properties and Enhances Antitumor Immunity in Head and Neck Squamous Cell Carcinoma. Cancer Immunol Res 2020;8:179-91.

12. Mezzadra R, Sun C, Jae LT, et al. Identification of CMTM6 and CMTM4 as PD-L1 protein regulators. Nature 2017;549:106-10.

13. Muranushi R, Araki K, Yokobori T, et al. High membrane expression of CMTM6 in hepatocellular carcinoma is associated with tumor recurrence. Cancer Sci 2021;112:3314-23.

14. Zheng Y, Wang C, Song A, et al. CMTM6 promotes cell proliferation and invasion in oral squamous cell carcinoma by interacting with NRP1. Am J Cancer Res;10:1691-709.

15. Guan X, Zhang C, Zhao J, et al. CMTM6 overexpression is associated with molecular and clinical characteristics of malignancy and predicts poor prognosis in gliomas. EBioMedicine 2018;35:233-43.

16. Zou Z, Tao T, Li H, et al. mTOR signaling pathway and mTOR inhibitors in cancer: progress and challenges. Cell Biosci 2020;10:31.

17. Gonzalez C, Sims JS, Hornstein N, et al. Ribosome profiling reveals a cell-type-specific translational landscape in brain tumors. J Neurosci 2014;34:10924-36. 
18. Chong DQ, Toh XY, Ho IA, et al. Combined treatment of Nimotuzumab and rapamycin is effective against temozolomide-resistant human gliomas regardless of the EGFR mutation status. BMC Cancer 2015;15:255.

19. Chen L, Zhai W, Zheng X, et al. Decreased IFIT2 Expression Promotes Gastric Cancer Progression and Predicts Poor Prognosis of the Patients. Cell Physiol Biochem 2018;45:15-25.

20. Liao YX, Zhang ZP, Zhao J, et al. Effects of Fibronectin 1 on Cell Proliferation, Senescence and Apoptosis of Human Glioma Cells Through the PI3K/AKT Signaling Pathway. Cell Physiol Biochem 2018;48:1382-96.

21. Wang K, Yang S, Gao Y, et al. MicroRNA-769-3p inhibits tumor progression in glioma by suppressing ZEB2 and inhibiting the $\mathrm{Wnt} / \beta$-catenin signaling pathway. Oncol Lett 2020;19:992-1000.

22. Nan Y, Guo H, Guo L, et al. MiRNA-451 Inhibits Glioma Cell Proliferation and Invasion Through the mTOR/HIF$1 \alpha /$ VEGF Signaling Pathway by Targeting CAB39. Hum Gene Ther Clin Dev 2018;29:156-66.

23. Kong F, Zhang W, Qiao L, et al. Establishment and quality evaluation of a glioma biobank in Beijing Tiantan Hospital. PeerJ 2018;6:e4450.

24. Delgado-López PD, Saiz-López P, Gargini R, et al. A comprehensive overview on the molecular biology of human glioma: what the clinician needs to know. Clin

Cite this article as: Wei L, Wei Q, Yang X, Zhou P. CMTM6 knockdown prevents glioma progression by inactivating the mTOR pathway. Ann Transl Med 2022;10(4):181. doi: 10.21037/atm-21-6894
Transl Oncol 2020;22:1909-22.

25. Burr ML, Sparbier CE, Chan YC, et al. CMTM6 maintains the expression of PD-L1 and regulates antitumour immunity. Nature 2017;549:101-5.

26. Imamovic D, Vranic S. Novel regulators of PD-L1 expression in cancer: CMTM6 and CMTM4-a new avenue to enhance the therapeutic benefits of immune checkpoint inhibitors. Ann Transl Med 2017;5:467.

27. Visan I. CMTM6 controls PD-L1. Nat Immunol 2017;18:1067.

28. Lisi L, Laudati E, Navarra P, et al. The mTOR kinase inhibitors polarize glioma-activated microglia to express a M1 phenotype. J Neuroinflammation 2014;11:125.

29. Ames HM, Yuan M, Vizcaíno MA, et al. MicroRNA profiling of low-grade glial and glioneuronal tumors shows an independent role for cluster 14q32.31 member miR487b. Mod Pathol 2017;30:204-16.

30. Chen H, Ma Z, Vanderwaal RP, et al. The mTOR inhibitor rapamycin suppresses DNA double-strand break repair. Radiat Res 2011;175:214-24.

31. Conciatori F, Bazzichetto C, Falcone I, et al. Role of mTOR Signaling in Tumor Microenvironment: An Overview. Int J Mol Sci 2018;19:2453.

32. Tian T, Li X, Zhang J. mTOR Signaling in Cancer and mTOR Inhibitors in Solid Tumor Targeting Therapy. Int J Mol Sci 2019;20:755. 